\title{
3D Tumor Segmentation from Volumetric Brain MR Images Using Level-Sets Method
}

\author{
Kamel Aloui and Mohamed Saber Naceur \\ National Engineering School, LTSIRS Laboratory - Tunis \\ Tunisia
}

\section{Introduction}

\subsection{Statement of the problem}

Segmentation in volumetric images is a tool allowing a diagnostics automation and as well will assist experts in quantitative and qualitative analysis. It's an important step in various applications such as visualization, morphometrics and image-guided surgery. In the context of neuro-imaging, brain tumor segmentation from Magnetic Resonance Images (MRI) is extremely important for treatment planning, therapy monitoring, examining efficacy of radiation and drug treatments and studying the difference between healthy subjects and subjects with brain tumor. The task of manually segmentation of brain tumor from MR images is generally time-consuming and difficult. Anyway, the task is done by marking by hand the tumor regions slice-by-slice which generates set of jaggy images, so the practitioner is confronted with a succession of boundary which he mentally stacked up to be made a 3D shape of brain tumor. This shape is inevitably subjective and becomes infeasible when dealing with large data sets, also there is losing of information in the third dimension because is not taken into account in the segmentation process. All this, affect the quality and accuracy of clinical diagnosis. An automatic or semi-automatic segmentation method of brain tumor that takes entire information within the volumetric MR image into account is desirable as it reduces the load on the human raters and generates optimal segmented images (Wang \& al., 2004), (Michael \& al., 2001), (Lynn \& al., 2001). Specially, automatic brain tumor segmentation presents many challenges and involves various disciplines such us pathology, MRI physics and image processing. Brain tumors are difficult to segment because they vary greatly in size and position, may be of any size, may have a variety of shapes and may have overlapping intensities with normal tissue and edema. This leads to numerous segmentation approaches of automatic brain tumor extraction. Low-level segmentation methods, such as pixel-based clustering, region growing, and filter-based edge detection, requires additional pre-processing and post-processing as well as considerable amounts of expert intervention and a priori knowledge on the regions of interest (ROI) (Sahoo \& al., 1988). Recently, several attempts have been made to apply deformable models to brain image analysis (Moon \& al., 2002). Indeed, deformable models refer to a large class of computer vision methods and have proved to be a successful segmentation technique for a wide range of applications. Deformable models, on the other hand, provide an explicit representation of the boundary and the ROI shape. They combine several desirable features such as inherent connectivity and smoothness, which counteract 
noise and boundary irregularities, as well as the ability to incorporate knowledge about the ROI. However, parametric deformable model must be re-parameterized dynamically to recover the object boundary and that has difficulty in dealing with topological adaptation such as splitting or merging model parts. A level-Sets deformable model, also referred to as a geometric deformable model, provides an elegant solution to address the primary limitations of parametric deformable models (Taheri \& al., 2009), (Taheri \& al., 2007), (Lefohn \& al., 2003). These methods have drawn a great deal of attention since their introduction in 1988. Advantages of the contour implicit formulation of the deformable model over parametric formulation include: no parameterization of the contour, topological flexibility, good numerical stability and straightforward extension of the $2 \mathrm{D}$ formulation to n-D.

\subsection{Outline of our method}

In this work, we describe various segmentation tools for segmenting brain tumor from volumetric MR images based on Level-Sets method. Figure 1 shows a general diagram of developed segmentation tools. We develop a first technique of brain tumor segmentation by stacking a sequence of 2D tumor contours, detected by 2D level-Sets method in the parallel cross-sectional MRI images. It consists on applying to each brain MRI slice the 2D level-Sets method and to propagate the result by taking as initial data the result of the preceding slice. The first approach is similar to an expert reasoning. This approach has several advantages such as simplicity to implement; it is fast, it requires less time than manual segmentation but it has a major disadvantage : the information loss because the third dimension is not taken into account. However, an evolution was necessary to the glance of its defects. The second and third developed approaches come to improve segmentation quality, based on carry out the computation in 3D space and detect the 3D tumor surface directly using 3D level-Sets method. In the second approach the 3D level-sets model evolves according to information related to contours on IRM volume. The third proposed method is similar to the segmentation with a deformable model with two phased image. Explicitly no need for gradient information, the level-Sets model evolves according to related regions information in the volumetric MR image.

In order to evaluate the proposed segmentation tools, MRI volumetric images have been used. They can be downloaded from the well known MeDEISA database "Medical Database for the Evaluation of Image and Signal Processing Algorithms », (MeDEISA, 2010).

\subsection{Structure of the chapter}

This chapter is organized as follows:

- In the next section, we present the level-Sets formulation and principal steps of the segmentation algorithm.

- In Section 3, 3D brain tumor segmentation by stacking a sequence of its 2D contours is described. For this purpose, 2D level-Sets method is required to detect tumor contours in the parallel cross-sectional MRI images.

- In section 4, we present 3D brain tumor segmentation using volumetric approaches based on boundary and region information on volumetric MRI data to check the deformable surface propagation.

- Finally, a summary and discussion related to this work are given in section 5 . 


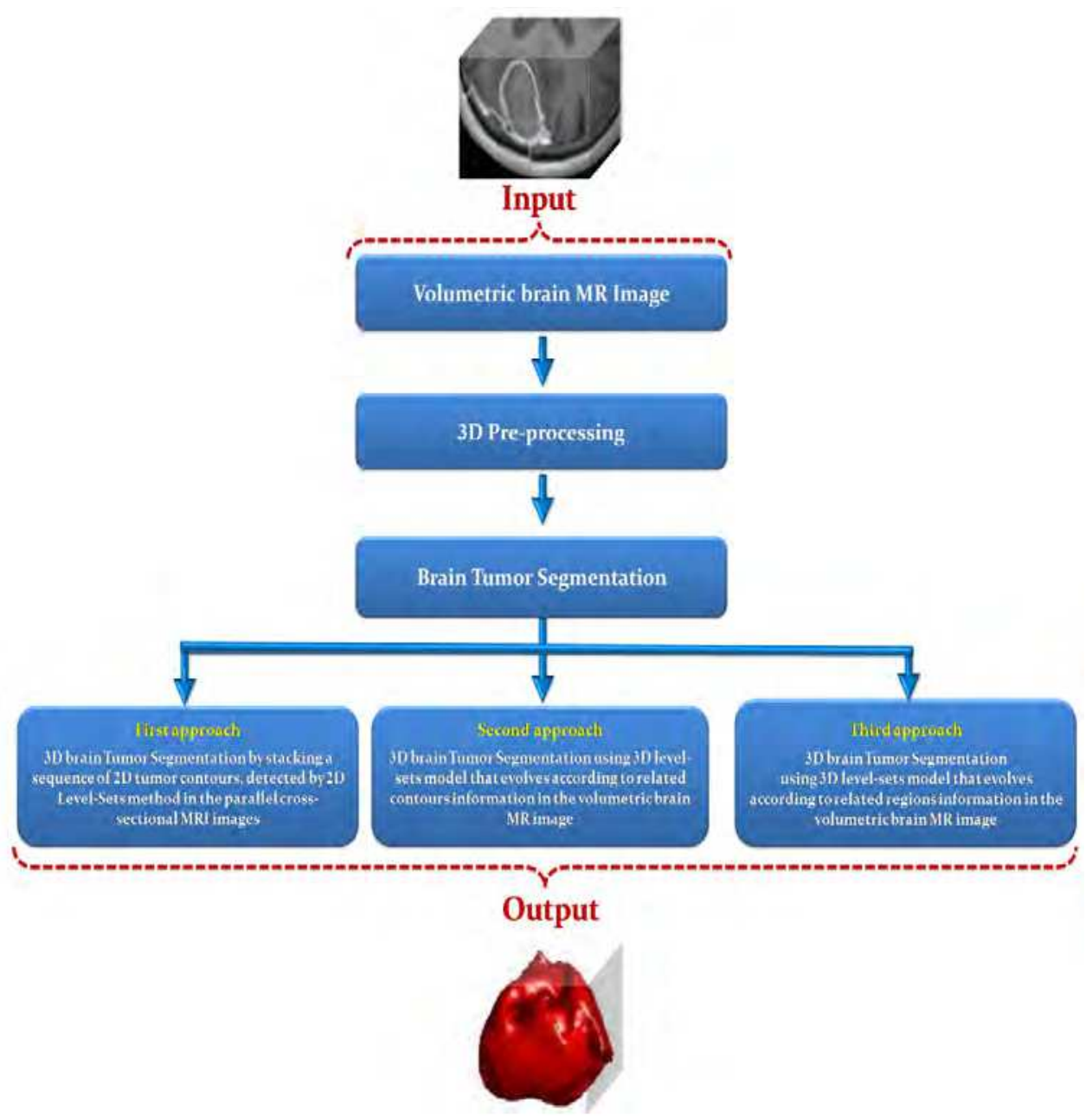

Fig. 1. General diagram of developed segmentation tools for segmenting 3D brain tumor from volumetric MR images based on Level-Sets method.

\section{Level-set method: Presentation}

In this Section, we describe a modeling technique based on a level-Sets approach for recovering shapes of objects in two and three dimensions. The modeling technique may be viewed as a form of active modeling such as "snakes" (Kass \& al., 1988) and deformable surfaces (Terzopoulos \& al. 1988). The model which consists of a moving front, until is plated on the desired shape, by externally applied stop criteria synthesized from the image data (Fig. 2.). Specially, deformable models are curves or surfaces defined in a digital image that can move under the influence of external and internal forces. External forces, which are computed from the image data, are designed to keep the model smooth during deformations. The external forces are defined from the deformable curve or surface like 
curvature in order to move the model to the boundary of a region of interest (ROI) in the digital image. Using these two forces, deformable models offer robustness to both image noise and boundary gaps, by constraining extracted ROI's boundaries to be smooth and incorporating other prior information about the ROI shape. Moreover, the resulting boundary representation can achieve subpixel accuracy which is considered a highly desirable property for medical imaging applications.

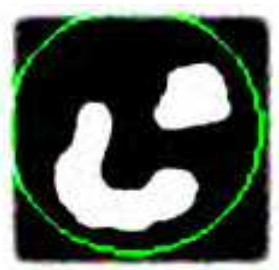

a)

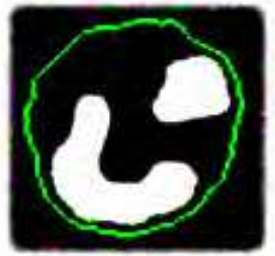

b)

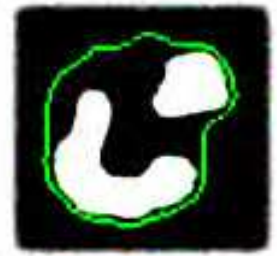

c)

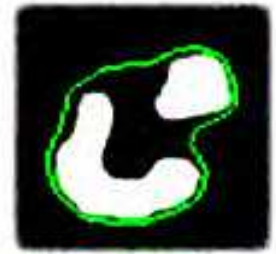

d)

Fig. 2. Principle of deformable curve in two space dimensions. We show curve evolution in time.

There are basically two types of deformable models:

- Parametric deformable models (Kass \& al., 1988), (Amini \& al., 1990), (Cohen, 1991) and (McInerney \& al., 1995);

- Geometric deformable models (Caselles \& al., 1993), (Milladi \& al., 1995), (Caselles \& al., 1995) and (Whitaker, 1994).

Parametric deformable models represent curves and surfaces explicitly in their parametric forms during deformation. This representation allows direct interaction with the model and can lead to a compact representation for fast real-time implementation. Adaptation of the model topology such as splitting or merging parts during the deformation can be difficult using parametric models. However, geometric deformable models can handle topological changes naturally. These models, based on the theory of curve evolution (Sapiro \& Tannenbaum, 1993), (Kimia \& al., 1995), (Kimmel \& al., 1995), (Alvarez \& al., 1993) and the level set method (Osher \& Sethian, 1988), (Sethian, 1999) represent curves and surfaces implicitly as a level-Sets of a higher-dimensional scalar function. Their parameterizations are computed only after complete deformation, thereby allowing topological adaptively to be easily accommodated. Despite this fundamental difference, the principles of both methods are very similar. Level-Sets method as a geometric deformable model; provide an elegant solution to address the primary limitations of parametric deformable models. In particular, curves and surfaces move using only geometric measures and other prior information from the image data to recover ROI boundaries.

In this section, we first review the fundamental concepts in curve evolution theory and the level-Sets method.

\subsection{Curve evolution theory}

The purpose of curve evolution theory is to study the deformation of curves using only geometric measures such as the curvature and the unit normal. Let us consider a moving curve $\Gamma(t)$, where $t$ represents the time;

The evolution of the curve along its normal direction can be characterized by the following partial differential equation (Fig. 3.): 


$$
\frac{\partial \Gamma}{\partial t}=F(k) \vec{N}
$$

Where:

- $\quad N$ denote inward unit normal;

- $k$ is the curvature.

Where $F$ is called speed function, since it determines the curve evolution speed. We note that a curve moving in some arbitrary direction can always be reparameterized to have the same form as Eq. 3 (Kimia, 1990). The intuition behind this fact is that the tangent deformation affects only the curve's parameterization, not its shape and geometry.

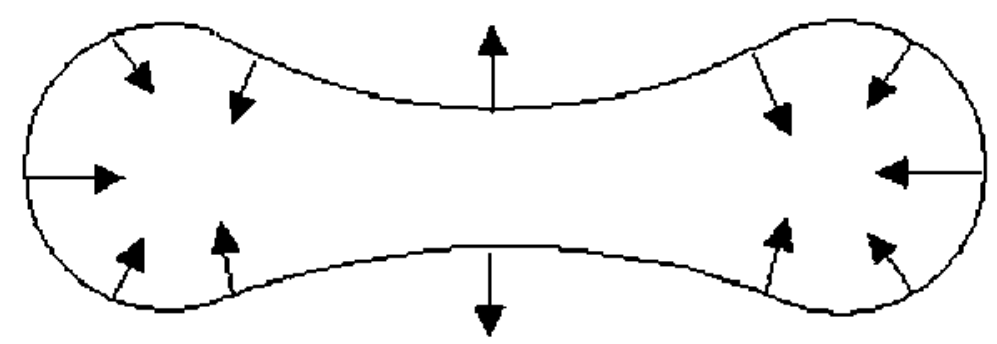

Fig. 3. Curve moving in the normal direction.

The most extensively studied curve deformations are constant deformation and curvature deformation. Constant deformation is given by:

$$
\frac{\partial \Gamma}{\partial t}=V_{0} \vec{N}
$$

Where $V_{0}$ is a coefficient determining the speed and direction of deformation (shrinks or expands). Constant deformation plays the same role as the pressure force in parametric deformable models.

Curvature deformation is given by the so-called geometric heat equation (Osher \& Sethian, 1988):

$$
\frac{\partial \Gamma}{\partial t}=\alpha k \vec{N}
$$

Where $\alpha$ is a positive constant. This equation will smooth a curve, eventually shrinking it to a circular point (Grayson, 1985). The use of the curvature deformation has an effect similar to the use of the elastic internal force in parametric deformable models.

The properties of curvature deformation and constant deformation are complementary to each other. Constant deformation can create singularities from an initially smooth curve while curvature deformation removes singularities by smoothing the curve.

The basic idea of the geometric deformable model is to couple the speed of deformation (using curvature and/or constant deformation) with the image data, so that the evolution of the curve stops at ROI's boundaries. The evolution is implemented using the level-Sets method. 


\subsection{Level-Sets method: Basic algorithms}

We now review the level-Sets method for implementing curve evolution. The level-Sets method is used to account for automatic topology adaptation, and it also provides the basis for a numerical scheme that is used by geometric deformable models. The level-Sets method for evolving curves is due to Osher and Sethian (Osher \& Sethian, 1988), (Sethian, 1985) and (Sethian, 1989). The interface $\Gamma$ bounds a (possibly multiply connected) region $\Omega$. The goal is to compute and analyze the subsequent motion of $\Gamma$ under a velocity field $F$. This velocity can depend on position $X$ (Where $X=(x, y)$ in tow space dimensions or $X=(x, y, z)$ in three space dimensions), time, the geometry of the interface and the external physics. The interface is captured as the zero level-Sets of a smooth function:

$$
\varphi(X, t) \text {, i.e., } \Gamma(t)=\{X \mid \varphi(X, t)=0\}
$$

$\varphi$ is positive inside $\Omega$, negative outside $\Omega$ and is zero on $\Gamma(t)$, has the following properties:

$$
\left\{\begin{array}{c}
\varphi(X, t) \succ 0 \quad \text { for } \quad X \in \Omega \\
\varphi(X, t) \prec 0 \quad \text { for } \quad X \notin \Omega \\
\varphi(X, t)=0 \quad \text { for } \quad X \quad \in \partial \Omega=\Gamma(T)
\end{array}\right.
$$

Thus, the interface is to be captured for all later time, by merely locating the set $\Gamma(t)$ for which $\varphi$ vanishes.

We note that the only purpose of the level-Sets function is to provide an implicit representation of the evolving curve and the topological merging and breaking are well defined and easily performed. Instead of tracking a curve through time, the level-Sets method evolves a curve by updating the level-Sets function at fixed coordinates through time. A useful property of this approach is that the level-Sets function remains a valid function while the embedded curve can change its topology. The motion is analyzed by convecting the $\varphi$ values (levels) with the velocity field $F$. This elementary equation is:

$$
\frac{\partial \varphi}{\partial t}+\vec{F}(k) \nabla \varphi=0
$$

Where $\nabla \varphi$ denotes the gradient of $\varphi$.

Here $F$ is the desired velocity on the interface, and is arbitrary elsewhere.

Actually, only the normal component of $F$ is needed. The inward unit normal to the levelSets curve is given by:

$$
\vec{N}=\frac{\nabla \varphi}{|\nabla \varphi|}
$$

Then

$$
F_{N}(k)=\vec{F}(k) \frac{\nabla \varphi}{|\nabla \varphi|}
$$


Accordingly, Eq. 8 becomes

$$
\frac{\partial \varphi}{\partial t}+F_{N}(k)|\nabla \varphi|=0
$$

Finally, the curvature $k$ at the zero level-Sets is given by:

$$
k=\operatorname{div}\left(\frac{\nabla \varphi}{|\nabla \varphi|}\right)=\frac{\varphi_{x x} \varphi_{y}^{2}-2 \varphi_{x} \varphi_{y} \varphi_{x y}+\varphi_{y y} \varphi_{x}^{2}}{\left(\varphi_{x}^{2}+\varphi_{y}^{2}\right)^{2 / 3}}
$$

\subsection{Level-sets: Speed function}

The geometric deformable contour formulation, proposed in (Caselles \& al., 1993) and (Malladi \& al., 1995), takes the following form:

$$
\frac{\partial \varphi}{\partial t}+F_{N}(k)|\nabla \varphi|=g(I)\left(V_{0}+\varepsilon k\right)|\nabla \varphi|
$$

Where

$$
g(I)=\frac{1}{1+|\nabla \hat{I}|^{p}}
$$

Positive $V_{0}$ shrinks the curve, and negative $V_{0}$ expands the curve. The curve evolution is coupled with the image data through a multiplicative stopping term $g(I)$. Where $\hat{I}$ is the image corrected by a Gaussian operator and $p=1$ or 2 . This scheme can work well for objects that have good contrast. However, when the object boundary is indistinct or has gaps, the geometric deformable contour may leak out because the multiplicative term only slows down the curve near the boundary rather than completely stopping the curve. Once the curve passes the boundary, it will not be pulled back to recover the correct boundary.

\subsection{Level-Sets: Numerical implementation}

Various numerical implementations of deformable models have been reported in the literature. For examples, the finite difference method (Kass \& al., 1988), dynamic programming (Amini \& al., 1990), and greedy algorithm (Williams \& Shah, 1992) have been used. In this section, we present the finite difference method implementation for level-Sets method as described in (Kass \& al., 1988).

\subsubsection{Initialization}

An initial function $\varphi(X, t=0)$ must be constructed such that its zero level-Sets correspond to the position of the initial contour or surface. A common choice is to set:

$$
\varphi(X, t=0)=d(x)
$$

Where $d(x)$ is the signed distance from each grid point to the zero level-Sets.

For example, when the zero level-Sets can be described by the exterior boundary of a circle, the signed distance function can be computed as follows: 


$$
\varphi(x, y, t=0)=\sqrt{\left(x-x_{0}\right)^{2}+\left(y-y_{0}\right)^{2}}-r
$$

Where $X_{0}=\left(x_{0}, y_{0}\right)$ is the center and $r$ is the radius of the circle.

\subsubsection{Discretization of the motion equation}

Since the motion equation Eq. 13 is derived for the zero level-Sets only, the speed function $F(k)$, in general, is not defined on other level-Sets. Hence, we need a method to extend the speed function $F(k)$ to all of the level-Sets. A re-initialization of the level-Sets function to a signed distance function is often required for level-Sets schemes.

The discretization of equation Eq. 13 is given as follows; noting $(i, j)$ is a position in the tow space dimensions image data:

$$
\frac{\varphi_{i j}^{n+1}-\varphi_{i j}^{n}}{\Delta t}=F_{i j}\left|\nabla_{i j} \varphi_{i j}^{n}\right|
$$

where :

- $\varphi_{i j}^{n}: \varphi$ values in position $(i, j)$ at the iteration $n \Delta t$.

- $\nabla_{i j} \varphi_{i j}^{n}$ : Spatial gradient approximation space of $\varphi_{i j}^{n}$ with the finite difference.

- $F_{i j}=g_{i j}(I)\left(F_{0 i j}+\varepsilon k_{i j}\right)$.

\subsubsection{Discretization of gradient}

If the temporal gradient approximation does not pose a problem, it is not the same for the spatial gradient. According to the spatial gradient is a factor in the curvature or constant term, it takes a different form (Osher \& Sethian, 1988), (Malladi \& al., 1995) and (Sethian, 1985). Indeed, if the curve evolves in various directions (eg according to its curvature), there is no particular problem. But, if the curve evolves in a given direction (eg. Constant deformation $V_{0}$ ), the choice of the spatial gradient is crucial: if it is calculated on a "simple", it can lead to loops formation during the deformation. Once the corner is developed, it is not clear how to continue the deformation, since the definition of the normal direction becomes ambiguous (Fig. 4.). A natural way to continue the deformation is to impose the so-called entropy condition originally proposed in the area of interface propagation by Sethian (Sethian, 1982), (Sethian, 1994).

Since the numerical scheme of the spatial gradient in one space dimension can be written as follows:

$$
\left|\nabla \varphi_{i}\right|=\left|\frac{\varphi_{i+1}-\varphi_{i-1}}{2 \Delta x}\right|
$$

When, there is a deformation in various directions, (when $\left|\nabla \varphi_{i}\right|$ is the curvature $k$ factor);

$$
\left|\nabla \varphi_{i}\right|=\left(\left(\max \left(D_{x}^{-} \varphi_{i}, 0\right)\right)^{2}+\left(\min \left(D_{x}^{+}, 0\right)\right)^{2}\right)^{1 / 2}
$$

When, there is a constant deformation, (when $\left|\nabla \varphi_{i}\right|$ is constant deformation $V_{0}$ factor); 
Where

- $D_{x}^{-} \varphi_{i}=\frac{\varphi_{i}-\varphi_{i-1}}{\Delta x}$

- $D_{x}^{+} \varphi_{i}=\frac{\varphi_{i+1}-\varphi_{i}}{\Delta x}$

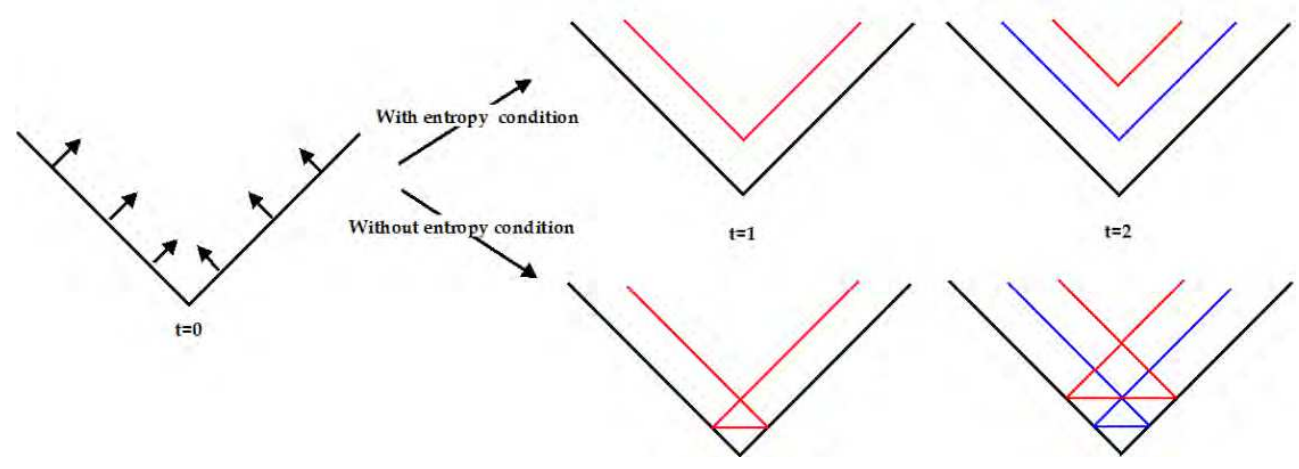

Fig. 4. Curve moving in the normal direction with the entropy condition. Deformation without entropy condition shows swallowtails and loops during the deformation.

\subsection{Numerical scheme of the motion function}

In this section, we provide a numerical implementation that is adapted from (Sethian, 1999) for Eq. 13, in which $\varepsilon$ and $V_{0}$ are allowed to be functions. The spatial derivatives are implemented using a special numerical scheme that can handle the formation of sharp corners during deformation. The numerical implementation is given as follows:

$$
\varphi_{i j}^{n+1}=\varphi_{i j}^{n}+\Delta t\left(\begin{array}{l}
g_{i j}(I) \varepsilon k_{i j}^{n}\left[\left(D_{i j}^{0 x}\right)^{2}+\left(D_{i j}^{0 y}\right)^{2}\right]^{1 / 2}+ \\
\max \left(g_{i j}(I) V_{0 i j}, 0\right) \nabla^{+} \varphi+\min \left(g_{i j}(I) V_{0 i j}, 0\right) \nabla^{-} \varphi
\end{array}\right)
$$

Where

$$
\begin{array}{ll}
D_{i j}^{0 x}=\frac{\varphi_{i+1, j}^{n}-\varphi_{i-1, j}^{n},}{2 \Delta x}, & D_{i j}^{0 y}=\frac{\varphi_{i, j+1}^{n}-\varphi_{i, j-1}^{n}}{2 \Delta x} \\
D_{i j}^{+x}=\frac{\varphi_{i+1, j}^{n}-\varphi_{i, j}^{n}}{\Delta x}, & D_{i j}^{+y}=\frac{\varphi_{i, j+1}^{n}-\varphi_{i, j}^{n}}{\Delta x} \\
D_{i j}^{-x}=\frac{\varphi_{i, j}^{n}-\varphi_{i-1, j}^{n}}{\Delta x}, & D_{i j}^{+y}=\frac{\varphi_{i, j}^{n}-\varphi_{i, j-1}^{n}}{\Delta x}
\end{array}
$$




$$
\begin{aligned}
& \nabla^{+} \varphi=\left[\begin{array}{l}
\left(\max \left(D_{i j}^{-x} \varphi_{i j}, 0\right)\right)^{2}+\left(\min \left(D_{i j}^{+x} \varphi_{i j}, 0\right)\right)^{2} \\
\left(\max \left(D_{i j}^{-y} \varphi_{i j}, 0\right)\right)^{2}+\left(\min \left(D_{i j}^{+y} \varphi_{i j}, 0\right)\right)^{2}
\end{array}\right]^{1 / 2} \\
& \nabla^{-} \varphi=\left[\begin{array}{l}
\left(\min \left(D_{i j}^{-x} \varphi_{i j}, 0\right)\right)^{2}+\left(\max \left(D_{i j}^{+x} \varphi_{i j}, 0\right)\right)^{2} \\
\left(\min \left(D_{i j}^{-y} \varphi_{i j}, 0\right)\right)^{2}+\left(\max \left(D_{i j}^{+y} \varphi_{i j}, 0\right)\right)^{2}
\end{array}\right]^{1 / 2}
\end{aligned}
$$

As it has been specified previously, the level-Sets function evolves using a speed function for the zero level-Sets only and using extended speed functions. Accordingly, the level-Sets can lose its property of being a signed distance function, causing inaccuracy in curvature and normal calculations. As a result, re-initialization of the level-Sets function to a signed distance function is often required for these schemes.

Usually, the distance map is reset using the following equation (Sussman \& al., 1997):

$$
\frac{\partial \varphi}{\partial t}=\operatorname{signe}(\varphi) \times(1-|\nabla \varphi|)
$$

Where

$$
\text { signe }(\varphi)=\left\{\begin{array}{ccc}
-1 & \text { si } & \varphi \prec-1 \\
|\varphi| & \text { si } & -1 \prec \varphi \prec 1 \\
+1 & \text { si } & \varphi \succ 1
\end{array}\right.
$$

Since the curve or the surface of ROI is recovred from the zero level-Sets only. We must therefore detect the zero values of the function $\varphi$.We can only detect differences in sign between two consecutive points in either direction, horizontal and vertical. The detection of points $P(i, j)$ of zero level-Sets by Malladi (Malladi \& al., 1995) is done according to the following algorithm:

Recovering interface algorithm:

Function isfront $(\varphi)$

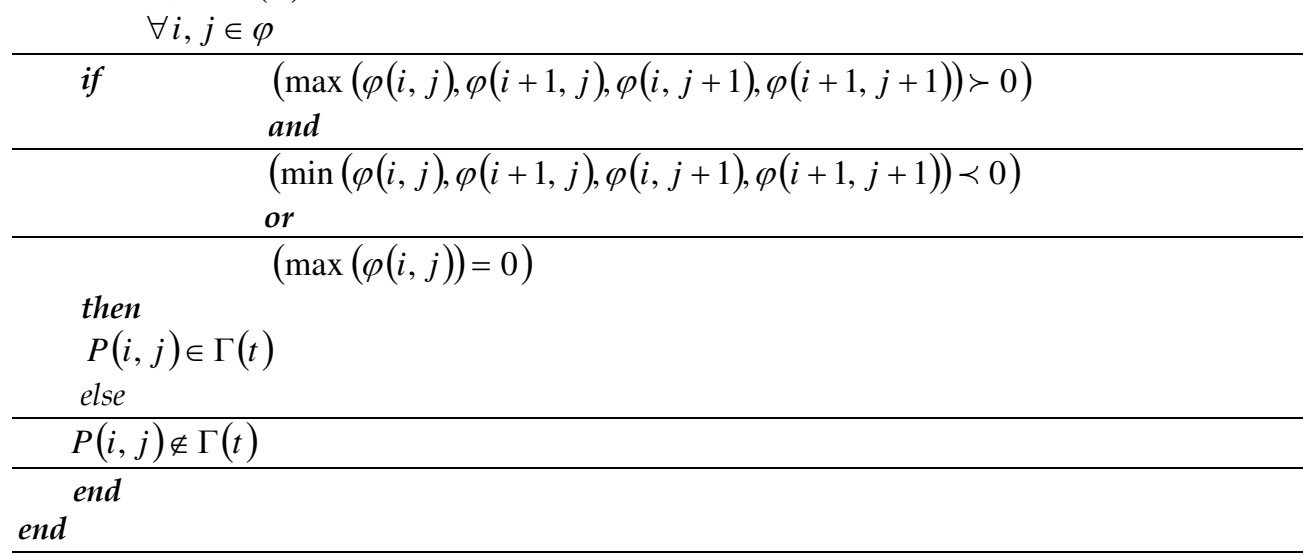




\subsection{Level-sets: Stop function}

The multiplicative stopping term of the speed function is defined as $g(I)$ given by eq. 14 . It has values that are closer to zero in regions of high image gradient and values that are closer to unity in regions with relatively constant intensity. $\hat{I}$ denotes the image convolved with a Gaussian smoothing filter whose characteristic width is $\sigma$. In some image slices, the boundary feature of the tumor is not salient enough and the image gradient information is weak. It usually causes the "boundary leaking" problem when we apply the level set method to detect the 3D tumor surface. The problem of the Gaussian filtering is the smoothing of the entire image, destroys and moves edges.

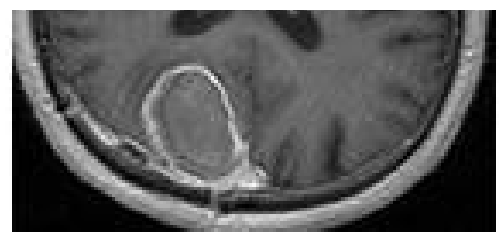

(a)

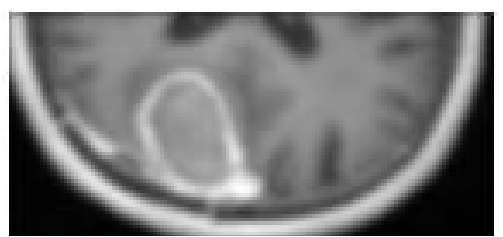

(b)

Fig. 5. Gaussian filters: destroys and moves edges. (a) Original image, (b) image smoothed by a Gaussian filter.

So we need to limit or prohibit the smoothing operation of the contours in the MR images. For this, we must choose a filter aiming at reducing image noise without removing significant parts of the image content, typically edges, lines or other details of the MR image. The anisotropic diffusion filter proposed by P. Perona and J. Malik (Perona \& Malik, 1990) meets our needs. The action of such filter is given by the following nonlinear equation:

$$
\frac{\partial I(X, t)}{\partial t}=\operatorname{div}(c(X, t) \cdot \nabla I(X, t))
$$

That preserves edges and only smooths regions with relatively constant intensity, $c(X, t)$, is called conduction coefficient. When the diffusion coefficient is chosen as an edge seeking function, the resulting equations encourage diffusion (hence smoothing) within regions with relatively constant intensity and prohibit it across strong edges. Hence, the edges can be preserved while removing noise from the image.

An illustration of the action of anisotropic diffusion filter is given by the following figure:

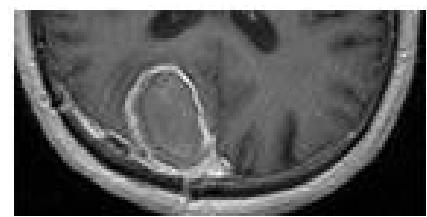

(a)

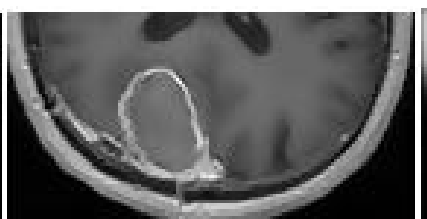

(b)

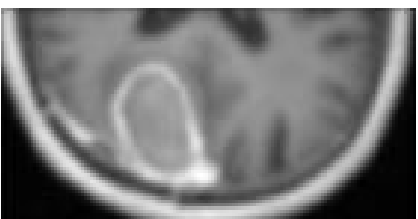

(c)

Fig. 6. Non linear diffusion filters: Preserves edges. (a) Original image, (b) image smoothed by a non linear diffusion filter. 
The following illustrations (Fig. ) show the variation of the stop function given by the equation 14, where we apply a Gaussian filter (a) and where we apply an anisotropic filtering (b).

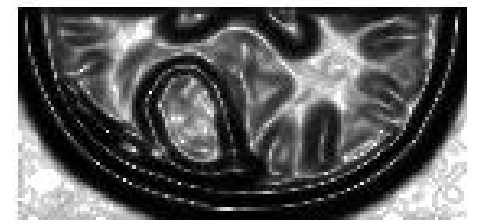

(a)

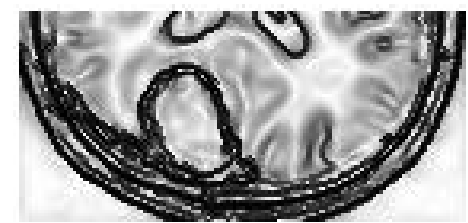

(b)

Fig. 7. Stop function $g(I)$. (a) Using Gaussian filter, (b) Using an anisotropic diffusion filter.

\section{3D tumor reconstruction from its $2 \mathrm{D}$ contours}

We develop a first technique of 3D brain tumor segmentation by stacking a sequence of 2D tumor contours, detected by 2D level-Sets method in the parallel cross-sectional MRI images (Fig. 7). It consists on applying to each brain MRI slice the level-Sets method in 2D and to propagate the result by taking as initial data the result of the preceding slice.

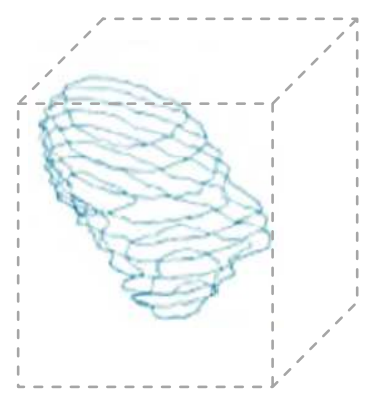

Fig. 8. 3D object reconstruction from its $2 \mathrm{D}$ contours.

The main stages of our algorithms are the following (Fig. 9.):

- Initialization of a curve around the tumor in the middle cross-sectional MRI images is called 'main slice' in this work.

- Run a level-Sets algorithm as it has been specified previously by the equation 20 , in the main slice.

- The brain tumor boundary in the main slice is used as initial curve in its tow contiguous slices (one upper slice and one lower slice) and so forth.

- The algorithm stops when all the cross-sectional MRI images are processed. After all tumor boundaries are stacked and 3D tumor shape is reconstructed.

The following figures (Fig. 10. and Fig. 11.) show various views of the surface of the tumor obtained by $3 \mathrm{D}$ reconstruction of its $2 \mathrm{D}$ contours and some projections of $2 \mathrm{D}$ tumor contours related of somes cross-sectional MRI images.

This approach is similar to an expert reasoning. This approach has several advantages such as simplicity to implement, it is fast, it requires less time than manual segmentation and based on 2D level-Sets method that has shown robustness in the segmentation of MRI images. 


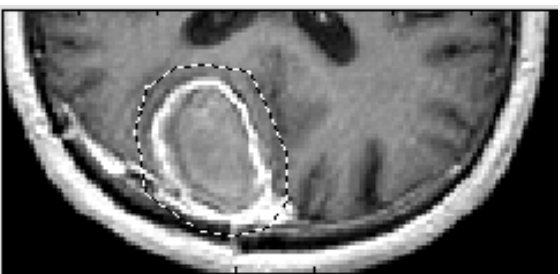

(a)

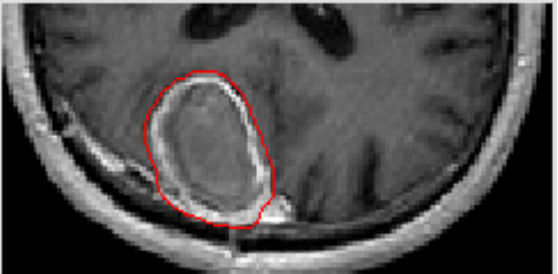

(b)

Fig. 9. Result of brain tumor segmentation in the middle MRI slice. (a) Initialization or initial curve, (b) final boundary of brain tumor.
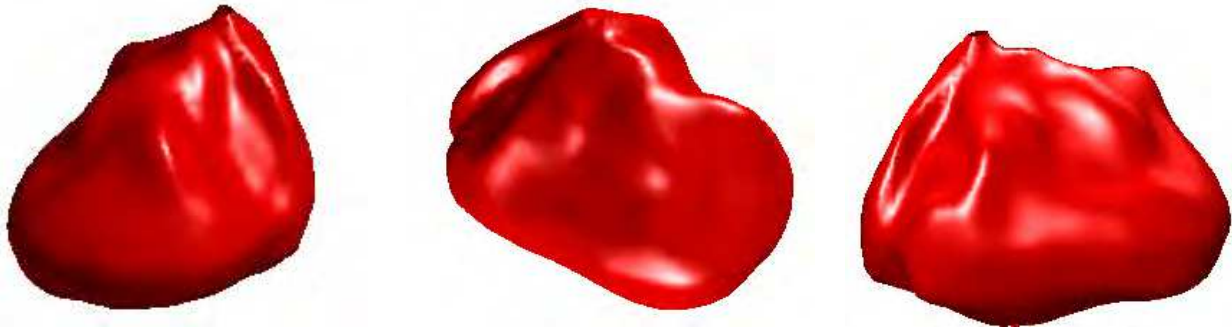

Fig. 10. 3D brain tumor surface visualization by stacking of its 2D boundaries.

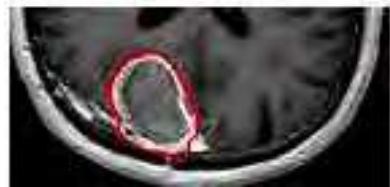

(a)

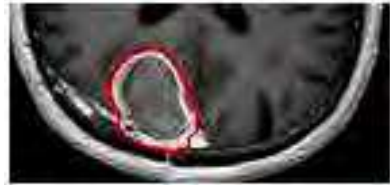

(b)

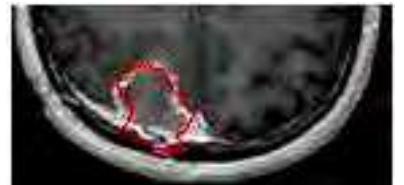

(c)

Fig. 11. Result of 2D segmentation: (a) slice 80, (b) slice 75, (c) slice 70.

\section{Volumetric approach}

Despite the advantages cited above related to segmentation based on stacking a sequence of 2D contours detected in the parallel cross-sectional images, it has many disadvantages: there is information loss because the third dimension is not taken into account, broken boundary in one slice and overlapping intensities usually lead to poor detected results. This approach supposes that the distance between the slices is very small and the reconstruction of the surface and its properties from 2D contours may lead to inaccurate results. However, an evolution was necessary to the glance of its defects. The following developed approaches come to improve segmentation quality, based on carrying out the computation in 3D space and detects the 3D tumor surface directly using 3D level-Sets method. First, the 3D level- 
Sets model evolves according to information related to contours on IRM volume specifically the data gradient information. Second, the level-Sets model evolves according to related regions information in the volumetric MR image.

\subsection{D level-Sets method with contours information}

The first stage of this method is to initialize a small sphere around the border of the brain tumor. Then the level-Sets model evolves according to information related to edges in the volumetric brain MR images. This movement comes to its end when the deformable surface found the actual border of the brain tumor.

3D discrete evolution equation of the level-Sets model is the following:

$$
\varphi_{i j k}^{n+1}=\varphi_{i j k}^{n}+\Delta t\left(\begin{array}{l}
g_{i j k}(I) \varepsilon k_{i j k}^{n}\left[\left(D_{i j k}^{0 x}\right)^{2}+\left(D_{i j k}^{0 y}\right)^{2}+\left(D_{i j k}^{0 z}\right)^{2}\right]^{1 / 2}+ \\
\max \left(g_{i j k}(I) V_{0 i j k}, 0\right) \nabla^{+} \varphi+\min \left(g_{i j k}(I) V_{0 i j k}, 0\right) \nabla^{-} \varphi
\end{array}\right)
$$

Where

$$
\begin{gathered}
\nabla^{+} \varphi=\left[\begin{array}{l}
\left(\max \left(D_{i j k}^{-x} \varphi_{i j k}, 0\right)\right)^{2}+\left(\min \left(D_{i j k}^{+x} \varphi_{i j k}, 0\right)\right)^{2} \\
\left(\max \left(D_{i j k}^{-y} \varphi_{i j k}, 0\right)\right)^{2}+\left(\min \left(D_{i j k}^{+y} \varphi_{i j k}, 0\right)\right)^{2} \\
\left(\max \left(D_{i j k}^{-z} \varphi_{i j}, 0\right)\right)^{2}+\left(\min \left(D_{i j k}^{+z} \varphi_{i j k}, 0\right)\right)^{2}
\end{array}\right]^{1 / 2} \\
\nabla^{-} \varphi=\left[\begin{array}{l}
\left(\min \left(D_{i j k}^{-x} \varphi_{i j k}, 0\right)\right)^{2}+\left(\max \left(D_{i j k}^{+x} \varphi_{i j k}, 0\right)\right)^{2} \\
\left(\min \left(D_{i j k}^{-y} \varphi_{i j k}, 0\right)\right)^{2}+\left(\max \left(D_{i j k}^{+y} \varphi_{i j k}, 0\right)\right)^{2} \\
\left(\min \left(D_{i j k}^{-z} \varphi_{i j}, 0\right)\right)^{2}+\left(\max \left(D_{i j k}^{+z} \varphi_{i j k}, 0\right)\right)^{2}
\end{array}\right]^{1 / 2}
\end{gathered}
$$

The curvature is given as follows:

$$
k=\frac{2 \varphi_{x} \varphi_{y} \varphi_{x y}-\varphi_{x} \varphi_{z} \varphi_{x z}+2 \varphi_{y} \varphi_{z} \varphi_{y z}}{\left(\varphi_{x}^{2}+\varphi_{y}^{2}+\varphi_{z}^{2}\right)^{2 / 3}}
$$

To stop the evolution of 3D level-sets model in the desired boundaries we used 3D version of the anisotropic diffusion filter in order to reduce noise without removing significant parts of the brain MRI volume and without evolving the deformable surface toward the brain tumor borders.

We show in the following figure the results of the 3D brain tumor surface reconstruction using 3D level-Sets based contour's information. The following figure shows different stages of evolution of the deformable surface until reaching the final surface of the tumor and some projections in 2D slices (Fig. 12. and Fig. 13.).

Segmentation of 3D tumor in MR Images using volumetric approach based on the level-Sets as the surface detection mechanism. We note that the main problem with this approach is related to leakage or overflow of the deformable surface in regions where overlapping intensities are present and that usually leads to poor detected results. 

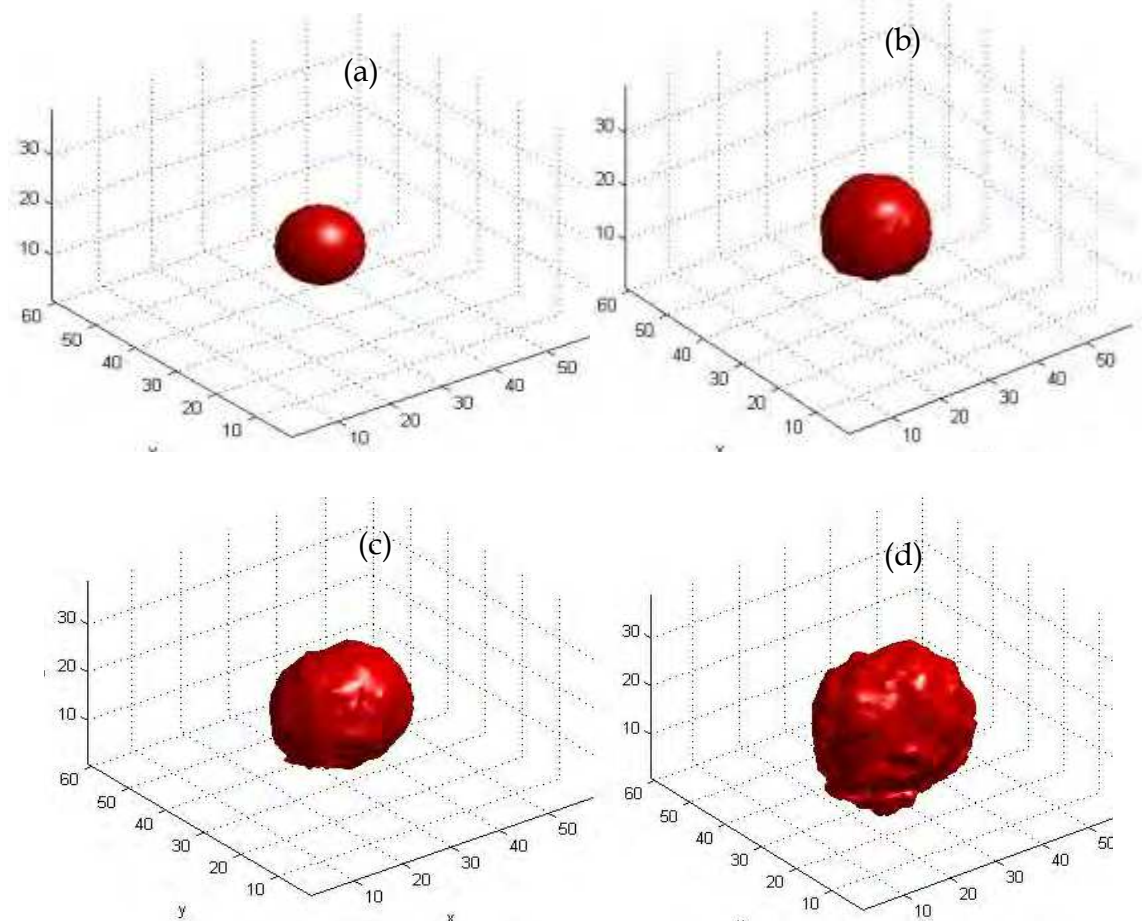

Fig. 12. 3D visualization of the surface of the brain tumor: (a) Initialization, (b) Iteration 10, (c) itiration 30, (d) iteration 50 .

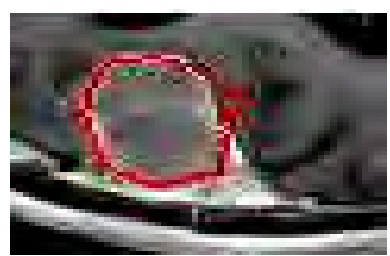

(a)

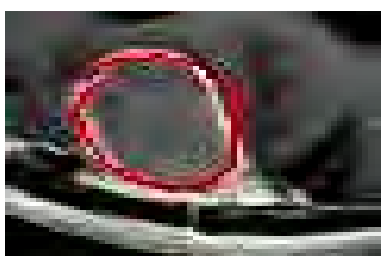

(b)

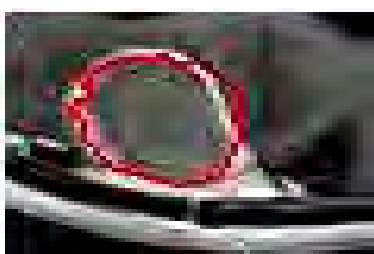

(c)

Fig. 13. 2D boundary visualization of the brain tumor: (a) slice 80, (b) slice 70, (c) slice 60.

\subsection{D level-Sets method with regions information}

In the previous approach,the segmentation quality is not good, it means that the gradient information which is local information insufficient to control the evolution of the level-Sets model. An alternative is to integrate statistical information related to regions in the brain MRI volume to improve the quality of brain tumor segmentation. Technically speaking, the new proposed method is similar to the segmentation with a deformable model with a two phased image (Chan \& Vese, 1999). The general principle is based on the evolution of a surface $\Gamma$ which partitions the volume data into several regions of different statistical characteristics. 
A single deformable surface $\Gamma$ allows segmentation into two regions $\Omega_{\text {in }}$ and $\Omega_{\text {out }}$, where $\Omega_{\text {in }}$ represents the region that circumscribed by the surface $\Gamma$ and $\Omega_{\text {out }}$ the outer region (Angelini, 2005). The information that controls the evolution of the the new level-Sets model is usually based on statistical modelling of the various region in the volumetric data.

We assume that the image $I(x, y, z)$ defined on the domain $\Omega$ is composed of two homogeneous regions of distinct values $I_{0}$ and $I_{1}$ and that the tumor region to detect corresponds to the region of intensity $I_{0}$. We denote the boundary of the tumor with intensity $I_{0}$ by $\Gamma$. For a given surface $\Gamma$ of the domain $\Omega$, we consider the following energy functional $E(\Gamma)$ :

$$
E(\Gamma)=\mu L(\Gamma)+v A(\Gamma)+\lambda_{0} \int_{\Omega_{\text {in }}}\left|I_{0}-c_{0}\right|^{2} d \Omega+\lambda_{0} \int_{\Omega_{\text {out }}}\left|I_{1}-c_{1}\right|^{2} d \Omega
$$

Where $c_{1}$ and $c_{2}$ are equal respectively to the average value of inside and outside of the surface $\Gamma \cdot L(\Gamma)$ and $A(\Gamma)$ are the regularizing terms corresponding respectively to the length of the curve and the area of the object enclosed by the curve.

$\mu, v, \lambda_{1}, \lambda_{2}$ are fixed positive parameters.

Segmentation of the brain tumor from volumetric MRI image is performed via minimization of the energy functional defined in Eq. (26). Minimization of the functional is proceeded using a steepest gradient descent on a discrete spatial grid indexed with $(i, j, k) \in \mathfrak{R}^{3}$ and introduction of a temporal index (n) leads to an iterative scheme with the following equation of the level-Sets evolution model:

$$
\varphi_{i j k}^{n+1}=\varphi_{i j k}^{n}+\nabla t \times \delta_{\varepsilon}\left(\varphi_{i j k}^{n}\right)\left(-\mu k \varphi_{i j k}^{n}+\lambda_{1}\left(I_{i j k}-c_{1} \varphi_{i j k}^{n}\right)^{2}+\lambda_{2}\left(I_{i j k}-c_{2} \varphi_{i j k}^{n}\right)^{2}\right)
$$

To segment the brain tumor using this approach (Fig. 14.), we initialized an initial surface through its boundary. Then this surface evolves until reaching the actual border of the tumor. Several criteria can be incorporated to stop the process of segmentation: when the area of the deformable surface becomes constant or the volume of the region bounded by the deformable surface becomes constant or Energy function $E(\Gamma)$ reaches its minimum value. The latter criterion is sufficient but it has a problem of computational cost. The convergence of the deformable surface to the tumor border implies that the area and the volume of deformable surface becomes constant. However, area and volume computational is less. For this, we used as a stopping condition, area and volume of the deformable surface at a time.

We present above a flowchart designed in our research to isolate a brain tumor using levelSets method based on region informations:

This method consists in initializing a small sphere through the border of the brain tumor. Then the level-Sets model evolves according to related region information in the image in order to plate itself on the surface of the tumor. We show in the following the results of the $3 \mathrm{D}$ reconstruction of the brain tumor surface relayed to this approach. The following figure shows different stages of evolution of the deformable surface until reaching the final surface of the tumor and some projections in 2D slices (Fig. 15. and Fig. 16.).

These results show that this approach combine the following advantages: arbitrary initialization of the object anywhere in the image, no need for gradient information, self adaptation for inward and outward local motion. 


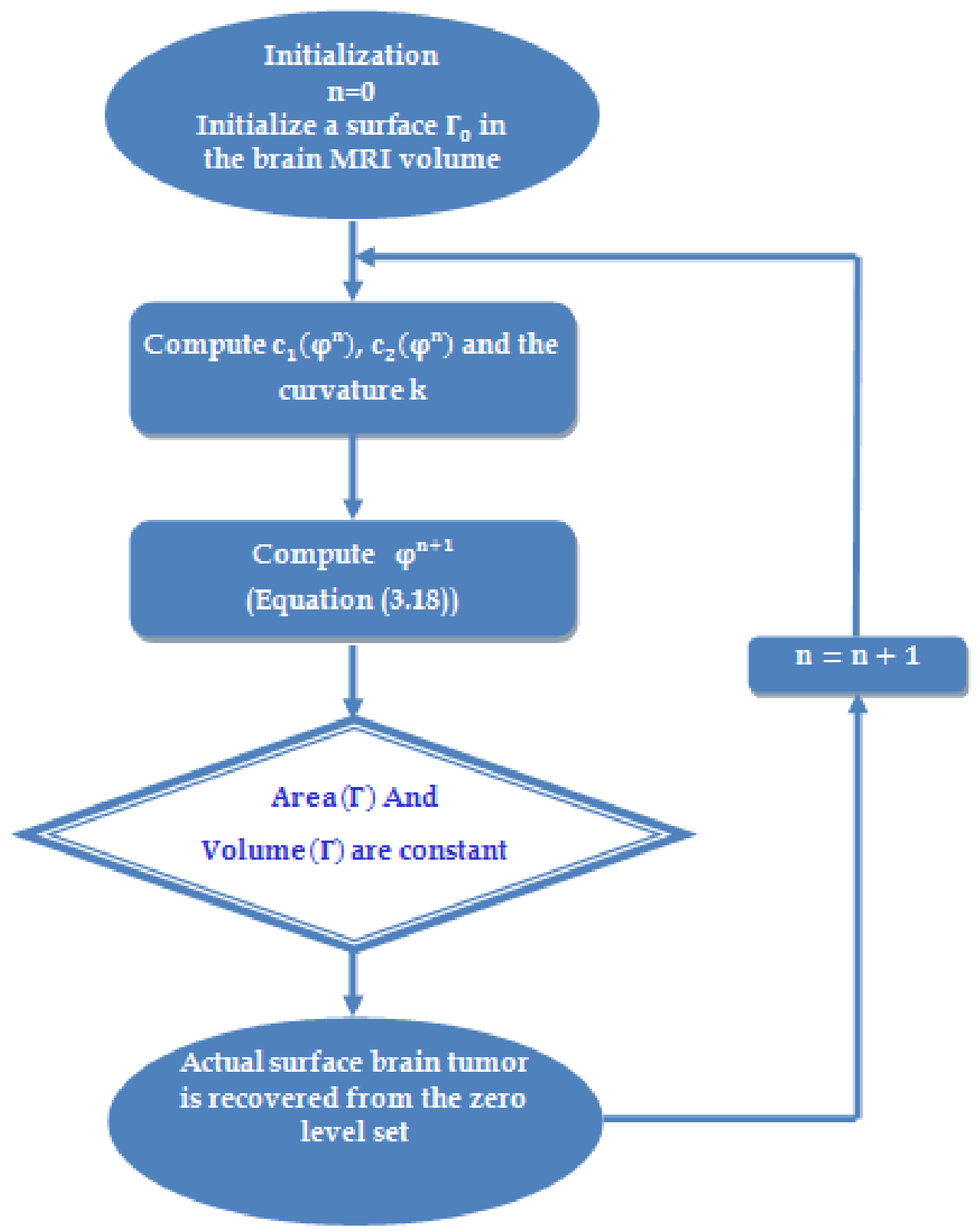

Fig. 14. Flowchart designed to isolate a brain tumor using the level-Sets method based on region informations. 

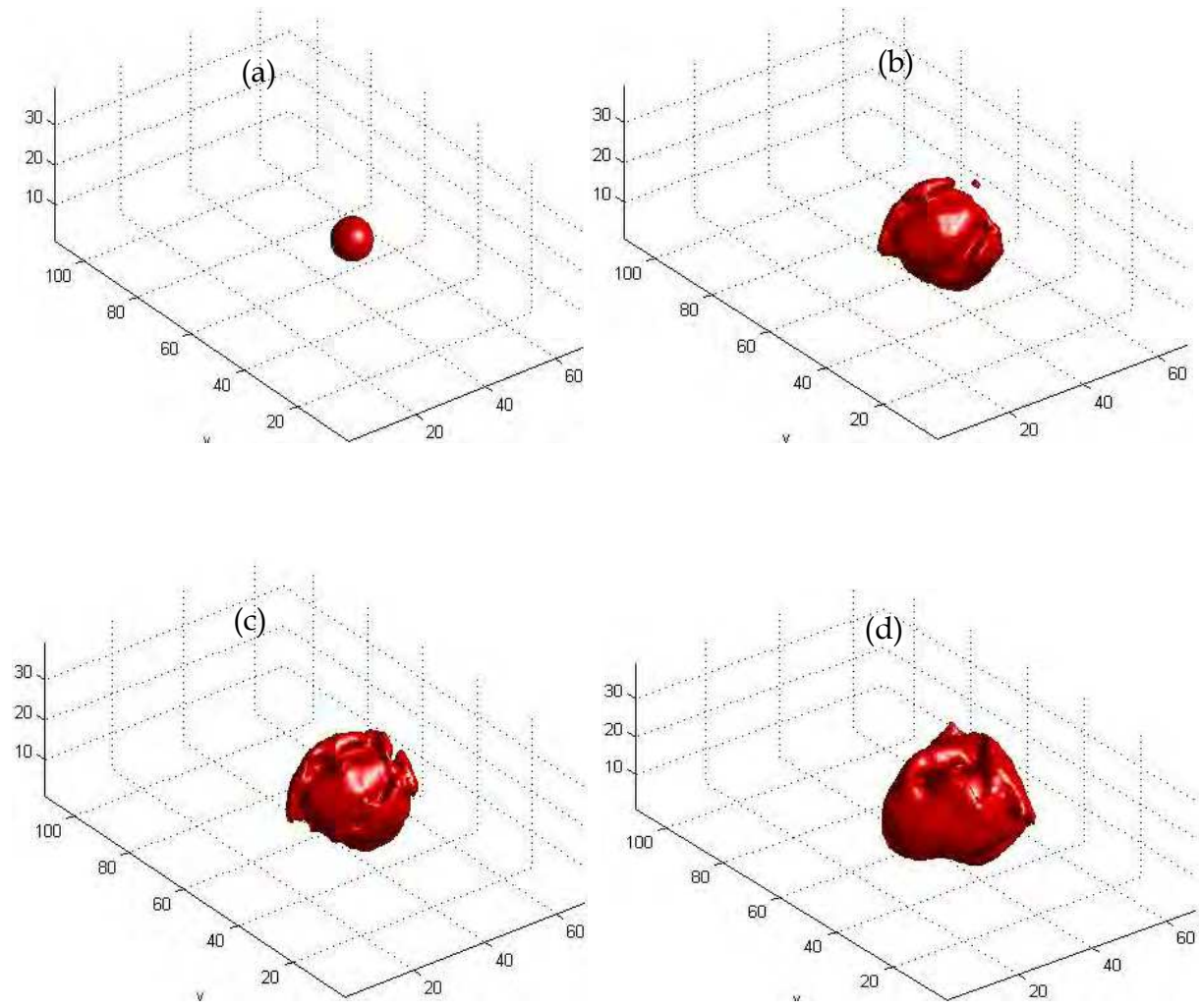

Fig. 15. 3D visualization of the surface of the brain tumor obtained by level-Sets method: (a) initialization, (b) iteration 80, (c) iteration 180, (d) iteration 300.

The following representation (Fig. 16) shows the segmentation result on some slicers.

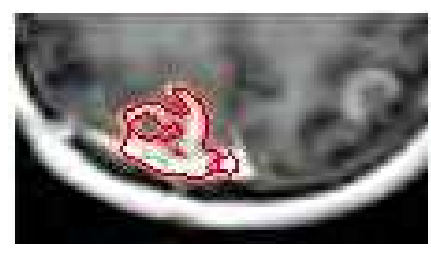

(a)

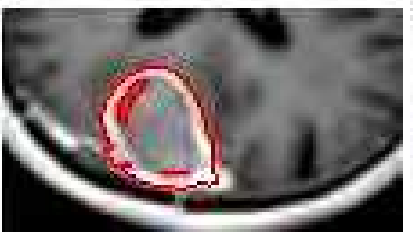

(b)

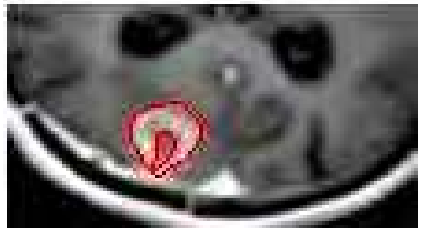

(c)

Fig. 16. Result of 2D segmentation: (a) slice 30, (b) slice 50, (c) slice 70. 


\section{Summary and discussion}

Presented research was provided with a general goal to develop 3D segmentation algorithms of brain tumor from volumetric MRI images. We have presented a variational method, 3D level-Sets applied to automatic segmentation of brain tumor in MRIs, using boundary and region based information of tumor to control the deformable surface propagation. The first approach used, is the $3 \mathrm{D}$ reconstruction from its $2 \mathrm{D}$ contours using a sequence of 2D contours, detected by 2D level-Sets method in the parallel cross-sectional MRI images. This method goes very well but it has two major defects, there is no interaction between the slices and surface must be cylindrical. This approach is the most simple that one can make. It makes it possible to use active contours in the field 2D method which showed its robustness. However, an evolution was necessary to the glance of its defects related to the results obtained and the tumor shapes that were being able to be treated. The second approach comes to improve the segmentation quality, based on carrying out the computation in 3D space and detecting the brain tumor region directly using 3D level-Sets method. In the first volumetric approach 3D level-Sets model evolves according to information related to contours on IRM volume. In the second level -Sets model evolves according to related regions descriptors in the volumetric MR image. Evaluations were performed on a set of volumetric MRI images obtained from (MeDEISA) database.

\section{References}

Kass, M.; Witkin, A. \& Terzopoulos, D. (1988). Snakes: Active contour models. International Journal of Computer Vision V. 1, no. , pp. 321-331, 1988.

Terzopoulos, D.; Witkin, A.; \& Kass, M. (1988). Constraints on deformable models: Recovering 3D shape and nonrigid motion. Artificial Intelligence, vol. 36, pp. 91. -1 23.1988 .

Amini, A.; Weymouth, T.; \& Jain, R. (1990). Using dynamic programming for solving variational problems in vision. IEEE Trans. Patt. Anal. Mach. Intell., vol. 12, no. 9, pp. 855-867, 1990.

Cohen, L. (1991). On active contour models and balloons. CVGIP: Imag. Under., vol. 53, no. 2, pp. 211-218, 1991.

McInerney, T. \& Terzopoulos, D. (1995). A dynamic finite element surface model for segmentation and tracking in multidimensional medical images with application to cardiac 4D image analysis. Comp. Med. Imag. Graph., vol. 19, no. 1, pp. 69-83, 1995.

Caselles, V.; Catte, F.; Coll, T. \& Dibos, F. (1993). A geometric model for active contours. Numerische Mathematik, vol. 66, pp. 1-31, 1993.

Malladi, R.; Sethian, J. \& Vemuri, B. (1995). Shape modeling with front propagation: A level set approach," IEEE Trans. Patt. Anal. Mach. Intell., vol. 17, no. 2, pp. 158$175,1995$.

Caselles, V.; Kimmel, R. \& Sapiro, G. (1995). Geodesic active contours. 5th International Conf. Comp. Vis., pp. 694-699, 1995. 
Whitaker, R. (1994). Volumetric deformable models: active blobs. Tech. Rep. ECRC-94-25, European Computer-Industry Research Centre GmbH, 1994.

Sapiro, G. \& Tannenbaum, A. (1993). Affine invariant scale-space," International Journal Computer Vision, vol. 11, no. 1, pp. 25-44, 1993.

Kimia, B.; Tannenbaum, A. \& Zucker, S. (1995). Shapes, shocks, and deformations: the components of two-dimensional shape and the reaction-diffusion space. International Journal Computer Vision. vol. 15, pp. 189-224, 1995.

Kimmel, R.; Amir, A. \& Bruckstein, M. (1995). Finding shortest paths on surfaces using level sets propagation. IEEE Trans. Patt. Anal. Mach. Intell., vol. 17, no. 6, pp. 635-640, 1995.

Alvarez, L.; Guichard, F.; Lions, P. \& Morel, J. (1993). Axioms and fundamental equations of image processing," Archive for Rational Mechanics and Analysis, vol. 123, no. 3, pp. 199-257, 1993.

Osher, S. \& Sethian, J. (1988). Fronts propagating with curvature-dependent speed: algorithms based on Hamilton-Jacobi formulations. J. Computational Physics, vol. 79, pp. 12-49, 1988.

Sethian, J. (1999). Level Set Methods and Fast Marching Methods: Evolving Interfaces in Computational Geometry, Fluid Mechanics. Computer Vision, and Material Science. Cambridge, UK: Cambridge University Press, 2nd ed., 1999.

Kimia, B. (1990). Conservation Laws and a Theory of Shape. Ph.D. thesis, McGill Centre for Intelligent Machines, McGill University, Montreal, Canada, 1990.

Grayson, M. (1985). Shortening embedded curves. Annals of Mathematics, vol. 129, pp. 71$111,1989$.

Sethian, J. (1985). Curvature and evolution of fronts. Commun. Math. Phys., vol. 101, pp. 487-499, 1985.

Sethian, J. (1989). A review of recent numerical algorithms for hypersurfaces moving with curvature dependent speed. J. Differential Geometry, vol. 31, pp. 131-161, 1989.

Williams, D. \& Shah, M. (1992). A fast algorithm for active contours and curvature estimation. CVGIP: Imag. Under., vol. 55, no. 1, pp. 14-26, 1992.

Malladi, R., Sethian, J. \& Vemuri, C. (1995). Shape Modeling with Front Propagation: A Level Set Approach, IEEE Trans. on Pattern Analysis and Machine Intelligence, 17, 2, pp. 158-175, 1995.

Sethian, J. (1982). An Analysis of Flame Propagation. Ph.D. thesis, Dept. of Mathematics, University of California, Berkeley, CA, 1982.

Sethian, J. (1994). Curvature flow and entropy conditions applied to grid generation.

Journal of Computational Physics, 115 : 440-454, 1994.

Sussman, M.; Fatemi, E.; Smereka, P. \& Osher, S. (1997). An improved level set method for incompressible two-phase flows. Computers and Fluids, vol. 27, 5-6, pp. 663-680, 1997. 
Perona, P. \& Malik, J. (1990). Scale-space and edge detection using anisotropic diffusion. IEEE Trans. Pattern Anal. Machine Intell., 12 :629.639, 1990.

Chan, T. \& Vese, L. (1999). An Active Contour model without Edges. In LNCS, edited by M. Neilsen, P. Johanson, O.F. Olson and J. Weickert, Springer-Verlag, Berlin/New York. Vol. 1687, 141-151,1999.

Angelini, E. (2005). Segmentation of Real-Time Three-Dimensional Ultrasound for Quantification of Ventricular Function: A Clinical Study on Right and Left Ventricles, Ultrasound in Med. \& Biol., Vol. 31, No. 9, pp. 1143-1158, 2005.

Sahoo, K.; Soltani, S.; Wong, C. \& Chen, Y. (1988). A survey of thresholding techniques. Computer Vision Graphics Image Processing 1988; 41: pp233 - 260.

Moon, N.; Bulitt, E.; Leemput, K. and Gerig, G (2002). Model-based brain and tumor segmentation. Proceedings of ICPR 2002; 1: 528-531.

Wang, Z.; Hu, Q.; Loe, K.; Aziz, A. \& Nowinski, L. (2004) Rapid and Automatic Detection of Brain Tumors in MR Images. Proceeding of SPIE 2004; 5369: 602 612.

Michael, K.; Simon, W.; Arya, N.; Peter, B.; Ferenc, J. \& Ron, K. (2001) Automated Segmentation of MR Images of Brain Tumors. Radiology 2001; 218: $586-591$.

Taheri, S.; Ong, S. \& Chong, V. (2009). Level-set Segmentation of Brain Tumors using a Threshold-based Speed Function," Image and Vision Computing (IVC) Elsevier Journal, 2009.

Taheri, S.; Ong, S. \& Chong, V. (2007). Threshold-based 3D Tumor Segmentation using Level Set (TSL). In Proc. IEEE Workshop on Application of Computer Vision (WACV), 2007.

Lefohn, A.; Cates, J. \& Whitaker, R. (2003). Interactive, GPU- Based Level Sets for 3D Brain Tumor Segmentation, MICCAI 2003.

Lynn, F.; Lawrence, H.; Dmitry, G. \& Murtagh, R. (2001). Automatic segmentation of nonenhancing brain tumors in magnetic resonance images. Artificial Intelligence in Medicine 2001; 21(1-3): pp $43-63$.

MeDEISA, (2010). Medical Database for the Evaluation of Image and Signal processing (MeDEISA), http://www.medeisa.net.

Lima, P.; Bonarini, A. \& Mataric, M. (2004). Application of Machine Learning, InTech, ISBN 978-953-7619-34-3, Vienna, Austria

Li, B.; Xu, Y. \& Choi, J. (1996). Applying Machine Learning Techniques, Proceedings of ASME 2010 4th International Conference on Energy Sustainability, pp. 14-17, ISBN 842-650823-3, Phoenix, Arizona, USA, May 17-22, 2010

Siegwart, R. (2001). Indirect Manipulation of a Sphere on a Flat Disk Using Force Information. International Journal of Advanced Robotic Systems, Vol.6, No.4, (December 2009), pp. 12-16, ISSN 1729-8806

Arai, T. \& Kragic, D. (1999). Variability of Wind and Wind Power, In: Wind Power, S.M. Muyeen, (Ed.), 289-321, Scyio, ISBN 978-953-7619-81-7, Vukovar, Croatia

Van der Linden, S. (June 2010). Integrating Wind Turbine Generators (WTG's) with Energy Storage, In: Wind Power, 17.06.2010, Available from 
http:/ / sciyo.com/articles/show/title/wind-power-integrating-wind-turbinegenerators-wtg-s-with-energy-storage 


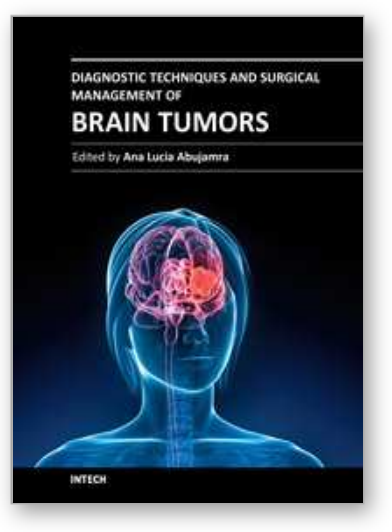

\author{
Diagnostic Techniques and Surgical Management of Brain Tumors \\ Edited by Dr. Ana Lucia Abujamra
}

ISBN 978-953-307-589-1

Hard cover, 544 pages

Publisher InTech

Published online 22, September, 2011

Published in print edition September, 2011

The focus of the book Diagnostic Techniques and Surgical Management of Brain Tumors is on describing the established and newly-arising techniques to diagnose central nervous system tumors, with a special focus on neuroimaging, followed by a discussion on the neurosurgical guidelines and techniques to manage and treat this disease. Each chapter in the Diagnostic Techniques and Surgical Management of Brain Tumors is authored by international experts with extensive experience in the areas covered.

\title{
How to reference
}

In order to correctly reference this scholarly work, feel free to copy and paste the following:

Kamel Aloui and Mohamed Saber Naceur (2011). 3D Tumor Segmentation from Volumetric Brain MR Images Using Level-Sets Method, Diagnostic Techniques and Surgical Management of Brain Tumors, Dr. Ana Lucia Abujamra (Ed.), ISBN: 978-953-307-589-1, InTech, Available from:

http://www.intechopen.com/books/diagnostic-techniques-and-surgical-management-of-brain-tumors/3d-tumorsegmentation-from-volumetric-brain-mr-images-using-level-sets-method

\section{INTECH}

open science | open minds

\section{InTech Europe}

University Campus STeP Ri

Slavka Krautzeka 83/A

51000 Rijeka, Croatia

Phone: +385 (51) 770447

Fax: +385 (51) 686166

www.intechopen.com

\section{InTech China}

Unit 405, Office Block, Hotel Equatorial Shanghai

No.65, Yan An Road (West), Shanghai, 200040, China

中国上海市延安西路65号上海国际贵都大饭店办公楼 405 单元

Phone: +86-21-62489820

Fax: +86-21-62489821 
(C) 2011 The Author(s). Licensee IntechOpen. This chapter is distributed under the terms of the Creative Commons Attribution-NonCommercialShareAlike-3.0 License, which permits use, distribution and reproduction for non-commercial purposes, provided the original is properly cited and derivative works building on this content are distributed under the same license. 